University of Nebraska - Lincoln

DigitalCommons@University of Nebraska - Lincoln

\title{
Computational Constitutive Model for Predicting Nonlinear Viscoelastic Damage and Fracture Failure of Asphalt Concrete Mixtures
}

\author{
Yong-Rak Kim \\ University of Nebraska - Lincoln, yong-rak.kim@unl.edu \\ D. H. Allen \\ University of Nebraska-Lincoln, dallen3@unl.edu \\ D. N. Little \\ Texas A\&M Univ., College Station, TX
}

Follow this and additional works at: https://digitalcommons.unl.edu/engineeringmechanicsfacpub

Part of the Mechanical Engineering Commons

Kim, Yong-Rak; Allen, D. H.; and Little, D. N., "Computational Constitutive Model for Predicting Nonlinear Viscoelastic Damage and Fracture Failure of Asphalt Concrete Mixtures" (2007). Faculty Publications from the Department of Engineering Mechanics. 45.

https://digitalcommons.unl.edu/engineeringmechanicsfacpub/45

This Article is brought to you for free and open access by the Mechanical \& Materials Engineering, Department of at DigitalCommons@University of Nebraska - Lincoln. It has been accepted for inclusion in Faculty Publications from the Department of Engineering Mechanics by an authorized administrator of DigitalCommons@University of Nebraska - Lincoln. 


\title{
Computational Constitutive Model for Predicting Nonlinear Viscoelastic Damage and Fracture Failure of Asphalt Concrete Mixtures
}

\author{
Yong-Rak Kim, M.ASCE,' D. H.Allen, ${ }^{2}$ and D. N. Little, F.ASCE ${ }^{3}$ \\ ' Assistant Professor, Department of Civil Engineering,W35I Nebraska Hall, \\ University of Nebraska-Lincoln, Lincoln, NE 68588-053I; Corresponding author — email ykim3@unl.edu \\ 2 Professor, Department of Engineering Mechanics, I 44 Othmer Hall, \\ University of Nebraska-Lincoln, Lincoln, NE 68588-0642; email dhallen@unlnotes.unl.edu \\ ${ }^{3}$ Professor, Department of Civil Engineering, 60I CE/TTI Building, \\ Texas A\&M University, College Station,TX 77843-3135; email d-little@tamu.edu
}

\begin{abstract}
A computational constitutive model was developed to predict damage and fracture failure of asphalt concrete mixtures. Complex heterogeneity and inelastic mechanical behavior are addressed by the model by using finite-element methods and elastic- viscoelastic constitutive relations. Damage evolution due to progressive cracking is represented by randomly oriented interface fracture, which is governed by a newly developed nonlinear viscoelastic cohesive zone model. Computational simulations demonstrate that damage evolution and failure of asphalt concrete mixtures is dependent on the mechanical properties of the mixture. This approach is suitable for the relative evaluation of asphalt concrete mixtures by simply employing material properties and fracture properties of mixture components rather than by performing expensive laboratory tests recursively, which are typically required for continuum damage mechanics modeling.
\end{abstract}

Keywords: asphalt concrete, constitutive models, viscoelasticity, damage, fracture, finite element method, mixtures

\section{Introduction}

Asphalt concrete mixtures are multiphased, particle-reinforced composites that consist of irregularly shaped and randomly oriented aggregate particles embedded in an inelastic matrix. Asphalt mixtures generally exhibit complicated mechanical behavior and multiple modes of damage. Although precise identification and prediction of the inelastic damage modes of the asphalt concretes is extremely difficult, it is important to seek out simpler approaches of predicting mechanical behavior including damage characteristics of the mixture in place of expensive and time-consuming laboratory experiments where possible.

One of the well-known modeling approaches is via the analytical/experimental continuum damage mechanics approach as presented in studies by Park et al. (1996), Lee et al. (2000, 2003), and many others (Daniel and Kim 2002; Kim et al. 2002; Masad et al. 2002; Chehab et al. 2003; Gibson et al. 2003; Tashman et al. 2004). Continuum damage models successfully predict structural degradation due to damage, but the models have limitations, since continuum damage modeling essentially ignores the heterogeneity in asphalt composites and hypothesizes that all damage in the asphalt sample can be averaged and represented by phenomenologically defined internal state variables called damage parameters. The damage parameters are phenomenologically determined by matching damage evolution characteristics with laboratory performance testing results typically through regression analyses. Therefore, the continuum damage mechanics approach requires that performance tests be performed wherever the mixture constituents are changed so that this approach may not reduce the amount of laboratory testing significantly.

Recently, computational modeling has been actively pursued as a means of overcoming the aforementioned shortcomings of continuum damage mechanics approaches, since the computational technique can account for the effect of mixture heterogeneity by dealing with mixture constituents separately. Many studies - such as by Guddati et al. (2002), Papagiannakis et al. (2002), Birgisson et al. (2002), Sadd et al. (2003), Soares et al. (2003) - have proposed computational techniques to characterize cracking performance of asphalt concrete mixtures, but they do not account for either material viscoelasticity or rate-dependent damage growth (including energy dissipation due to cracking), which is a typical phenomenon in asphalt concrete mixtures.

In order to model viscoelastic behavior including rate-dependent damage evolution, an in-house three-dimensional finite-element program was developed in this study. Material heterogeneity, geometric effects due to aggregate shape, and inelastic mechanical behavior of the asphalt concrete mixtures were incorporated in the finite-element model. The model can simulate crack-associated interface fracture within the asphalt mastic and along aggregate/asphalt boundaries. Interface fracture was modeled using a newly developed nonlinear viscoelastic cohesive zone model (Allen and Searcy 2000, 2001a,b). Progressive cracking and corresponding rate-dependent damage growth are thus included in the model.

Specific objectives of this study are:

- Develop a computational constitutive model capable of 


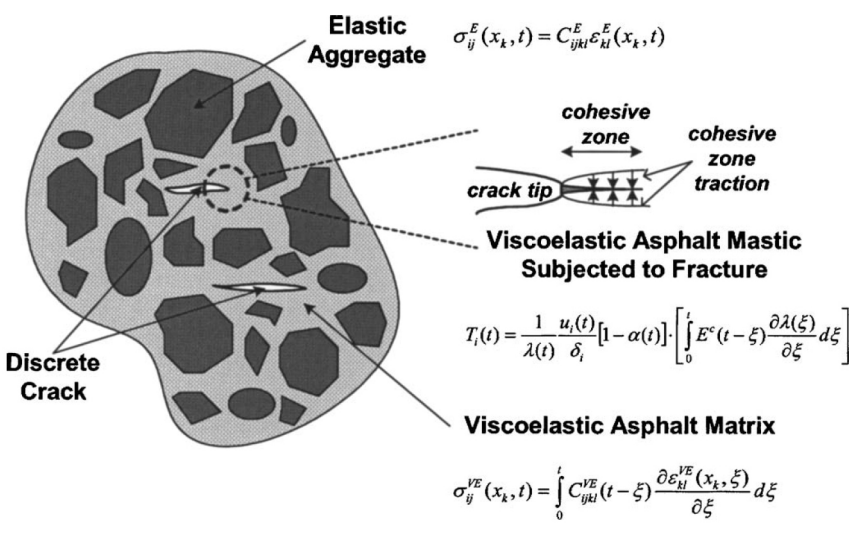

Figure I. General heterogeneous elastic-viscoelastic body containing discrete cracks and crack propagation via cohesive zone model.

predicting material viscoelasticity and fracture damage of heterogeneous asphalt composite mixtures;

- Evaluate rate-dependent damage behavior of asphalt concrete mixtures by implementing a micromechanics-based nonlinear viscoelastic cohesive zone model into the computational framework;

- Establish laboratory testing protocols to obtain model input, including fundamental material properties (elastic and viscoelastic) and a fracture-based probabilistic damage evolution function; and

- Evaluate predictive accuracy and efficiency of the computational model by comparing model simulations to laboratory testing data and by performing property-dependent mechanical analyses.

\section{Elastic-Viscoelastic Model Including Damage}

Figure 1 is a schematic view of a general heterogeneous elastic- viscoelastic body containing discrete cracks and potential paths of crack propagation. Assuming that the body represents asphalt concrete at room temperature, constitutive relations within the body can be simply represented by combining an elastic model of the coarse aggregate particles and a viscoelastic model of the asphalt matrix that is composed of asphalt mastic (asphalt cement mixed with a filler), fine aggregate particles, and air voids. The linear elastic constitutive relationship for the coarse aggregate particles can be expressed as

$$
\sigma_{i j}^{E}\left(x_{k^{\prime}}, t\right)=C_{i j k l}^{E} \varepsilon_{k l}^{E}\left(x_{k^{\prime}} t\right) \text { in volume } V_{E}
$$

where $\sigma_{i j}^{E}\left(x_{k}, t\right)=$ elastic stress as a function of time and space; $\varepsilon_{k l}^{E}\left(x_{k^{\prime}}, t\right)=$ elastic strain as a function of time and space; $C_{i j k l}^{E}$ $=$ elastic modulus which is constant; $x_{k}=$ spatial coordinates within the body; $t=$ time of interest; and $V_{E}=$ volume fraction of elastic aggregates.

Similarly, the constitutive behavior of the asphalt matrix can often be represented by the following linear viscoelastic (VE) convolution integral:

$$
\sigma_{i j}^{\mathrm{VE}}\left(x_{k}, t\right)=\int_{0}^{t} C_{i j k l}^{\mathrm{VE}}(t-\xi) \frac{\partial \varepsilon_{k l}^{\mathrm{VE}}\left(x_{k}, \xi\right)}{\partial \xi} d \xi \text { in volume } V_{\mathrm{VE}}
$$

where $\sigma_{\mathrm{v} \ddot{\mathrm{B}}}^{\mathrm{VE}}\left(x_{k^{\prime}} t\right)=$ viscoelastic stress as a function of time and space; $\varepsilon^{\mathrm{VE}}\left(x_{k^{\prime}}, t\right)=$ viscoelastic strain as a function of time and space; $C_{i j k l}^{k \mathrm{k}}=$ stress relaxation modulus which is time-depen- dent; $\xi=$ time-history integration variable; and $V_{\mathrm{VE}}=$ volume fraction of viscoelastic asphalt matrix.

The process or damage zone that precedes the crack tip reflects the constitutive behavior of the material in the damage zone caused by small-scale damage accumulation. This behavior can be modeled in many different ways, and one of the well-known approaches is to use a cohesive zone, as illustrated in Figure 1. Cohesive zone models are well-established tools in classical fracture mechanics that have been developed to remove stress singularities ahead of crack tips and to improve predictions of crack tip advancement in viscoelastic media.

The concept of a cohesive zone was first proposed by Dugdale (1960) and Barenblatt (1962). They postulated that there are certain regions preceding crack tips where nonzero tractions could be specified to inhibit crack tip advancement. However, Dugdale's model is not applicable to a broad range of materials, since the cohesive zone model is defined by constant traction ahead of the crack tip with a value equivalent to that of the yield stress of the surrounding bulk material. Most materials demonstrate nonlinear and inelastic complex traction-displacement behavior at the crack tip. In 1987, Needleman used a potential function and developed a constitutive model for a cohesive zone in which the tractions were described in terms of the normal displacement difference across the cohesive interface. Following Needleman's work, Tvergaard (1990) developed a cohesive zone model in the form of a cubic traction-displacement relation based on a nondimensional displacement parameter with decohesion that is dependent upon both normal and tangential interfacial displacement differences. The cohesive zone models by Needleman (1987) and/or Tvergaard (1990) can predict the development of new surface areas in the body due to cracking, but they are somewhat inappropriate for modeling crack growth and fracture failure of asphalt concretetype mixtures, since the Needleman and Tvergaard models are neither history dependent nor rate dependent. It is well established that asphalt mixtures are time-, history-, and rate-dependent viscoelastic. Damage evolution behavior of the asphalt mixtures is extremely complicated. Consequently, an analytical approach to solve the viscoelastic problem associated with crack growth is perhaps not feasible.

In an attempt to simulate damage growth due to cracks in viscoelastic media, Allen and Searcy $(2000,2001 a, b)$ recently formulated a nonlinear, viscoelastic cohesive zone model based on micromechanics. This model can reflect nonlinear viscoelastic damage growth and is appropriate for predicting damage evolution, corresponding material softening, and eventual fracture failure of highly inelastic asphalt concrete mixtures. Therefore, the writers selected the Allen and Searcy model to simulate damage growth. The three-dimensional traction-displacement relationship for the nonlinear viscoelastic cohesive zone (CZ) model is as follows (Allen and Searcy 2000, 2001a):

$$
\begin{gathered}
T_{i}(t)=\frac{1}{\lambda(t)} \frac{u_{i}(t)}{\delta_{i}}[1-\alpha(t)]\left[\int_{0}^{t} E^{c}(t-\xi) \frac{\partial \lambda(\xi)}{\partial \xi} d \xi\right] \\
\text { on boundary } \partial V_{\mathrm{CZ}}
\end{gathered}
$$

where $i=n$ (normal direction), $t$ (tangential direction), or $r$ (radial direction); $T_{i}=$ cohesive zone traction; $u_{i}=$ cohesive zone displacement; $\delta_{i}=$ cohesive zone material length parameter; $\lambda(t)=$ cohesive zone strain $=\left\{\left[u_{n}(t) / \delta_{n}\right]^{2}+\left[u_{t}(t) / \delta_{t}\right]^{2}+\left[u_{r}(t) /\right.\right.$ $\left.\left.\delta_{r}\right]^{2}\right\}^{1 / 2} ; \alpha(t)=$ microscale damage evolution function; $E^{c}(t)=$ stress relaxation modulus of the cohesive zone; and $\partial V_{\mathrm{CZ}}=$ internal boundary representing cohesive zone. The material 


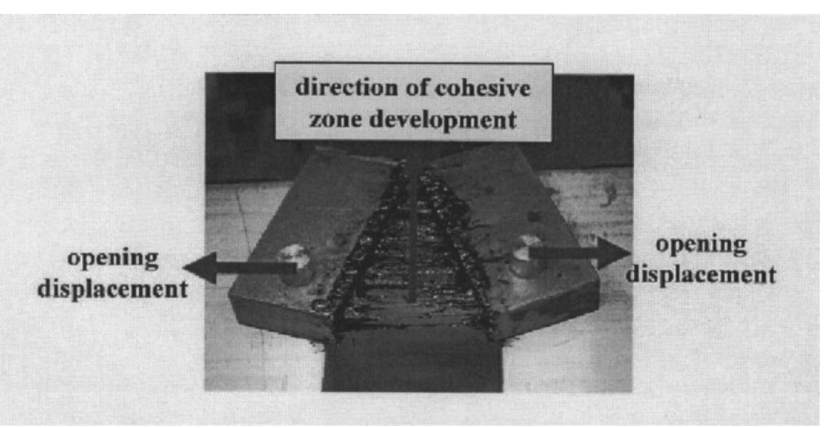

Figure 2. Schematic of fracture testing to define cohesive zone fracture properties.

length parameter $\left(\delta_{i}\right)$ in the cohesive zone model [Equation (3)] typically reflects a length scale intrinsic to the scale of the damaged zone. The length scale parameters are often considered critical relative displacements in the cohesive zone when the complete separation of the cohesive zone occurs. Progressive nonlinear damage is characterized by the damage evolution function $\alpha(t)$. When $\alpha(t)$ reaches the value of unity in Equation (3), the crack face traction decays to zero, thus resulting in crack extension.

The damage evolution function can be experimentally determined by performing small-scale fracture tests to represent a locally averaged cross-sectional area of damaged material in a cohesive zone. In 2001, Williams developed such a testing method that monitors crack tip propagation represented by variation of fibril geometry in the cohesive zone. Figure 2 is a schematic of the fracture testing protocol developed by Williams (2001). As can be seen in Figure 2, continuous fibril development and cohesive zone growth due to damage are captured using image techniques, and geometric variation of the material fibrils (length, size, and width of each material fibril) is monitored by pixel counts in the image analysis. By assuming the geometry of each fibril as a right circular cylinder, and that the distribution of fibril radii in a whole cohesive zone is governed by a normal distribution, the probabilistic distribution of fibril radii can be expressed by (Allen and Searcy 2001b)

$$
f[r(t), \zeta]=\frac{1}{\zeta \sqrt{2 \pi}} \exp \left[\frac{-\left\{r-r^{o}[1-\nu \lambda(t)]\right\}^{2}}{2 \zeta^{2}}\right]
$$

where $f[r(t), \zeta]=$ normal distribution function of fibril radii; $r(t)=$ average value of fibril radii at time $t ; \zeta=$ standard deviation of fibril radii (assumed to be independent of time); $r^{0}=a v-$ erage value of initial fibril radii; and $v=$ Poisson's ratio of fibrils (assumed to be independent of time). Figure 3 illustrates a graphical depiction of Equation (4). As damage evolves, the average fibril radius and distribution curve correspondingly move to the left, because all fibrils become thinner. The distribution curve eventually passes a boundary that is marked as a critical fibril radius, $r_{\mathrm{cr}}$. The area under the distribution curve and left of the critical fibril radius represents the measure of fibril breakage or damage. This leads to the following expression for the experimentally determined damage evolution function (Allen and Searcy 2001b)

$$
\alpha(t)=1-\frac{1}{\zeta \sqrt{2 \pi}} \int_{r_{\mathrm{cr}}}^{\infty} \exp \left[\frac{-\left\{r-r^{o}[1-v \lambda(t)]\right\}^{2}}{2 \zeta^{2}}\right] d r
$$

where $r_{\mathrm{cr}}=$ average value of critical fibril radii.

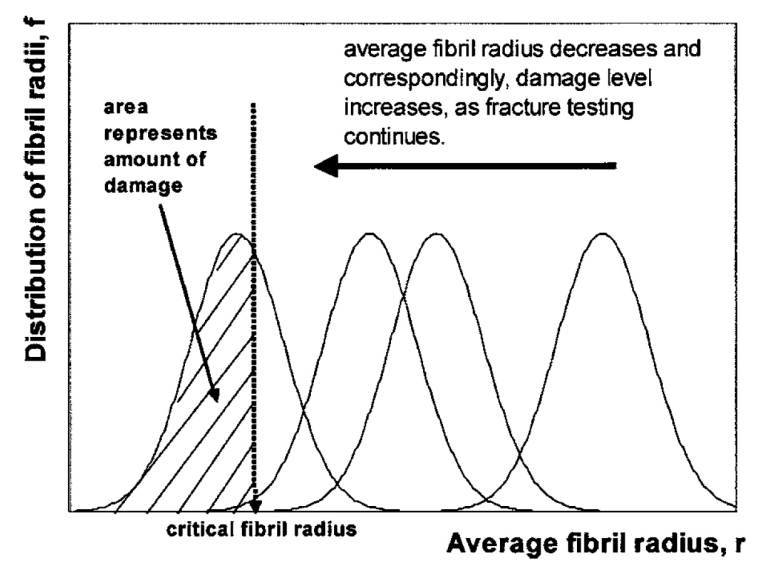

Figure 3. Probabilistic distribution of fibril radii.

\section{Finite-Element Formulation}

In Equations (2) and (3), the viscoelastic constitutions for the asphalt matrix phase and cohesive zones are expressed as time- and history-dependent convolution integrals. In an attempt to place the viscoelastic constitution into a computational finite-element model, it must be incrementalized so that the history dependence is retained at each time step. A more detailed description of the numerical implementation for viscoelastic media can be found in a study by Zocher et al. (1997).

Assuming that primary state variables (stress, strain, and displacement) at time $t$ are known, and the state variables at time $t+\Delta t$ are evaluated, the finite-element model can be formulated based on a time-incrementalized numerical scheme. Applying the strain-displacement operator, $\{\Delta \varepsilon\}=[B]\{\Delta u\}$, and the displacement-shape function relation, $\{\Delta u\}=[N]\{\Delta U\}$, the resulting finite-element formulation for the elastic-viscoelastic asphalt concrete sample including cohesive zone fracture is presented in matrix form as

$$
\left[K^{e}\right]\left\{\Delta U^{e}\right\}=\left\{f_{1}^{e}\right\}+\left\{f_{2}^{e}\right\}+\left\{f_{3}^{e}\right\}+\left\{f_{4}^{e}\right\}+\left\{f_{5}^{e}\right\}
$$

where

$$
\begin{gathered}
{\left[K^{e}\right]=\int_{V}[B]^{T}[C][B] d V+\int_{S_{c}}[N]^{T}[k][N] d S} \\
\left\{f_{1}^{e}\right\}=\int_{S_{e}}[N]^{T}[T(t+\Delta t)] d S \\
\left\{f_{2}^{e}\right\}=-\int_{V}[B]^{T}[\sigma(t)] d V \\
\left\{f_{3}^{e}\right\}=-\int_{V}[B]^{T}\left[\Delta \sigma^{R}\right] d V \\
\left\{f_{4}^{e}\right\}=-\int_{S_{C}}[N]^{T}[T(t)] d S
\end{gathered}
$$



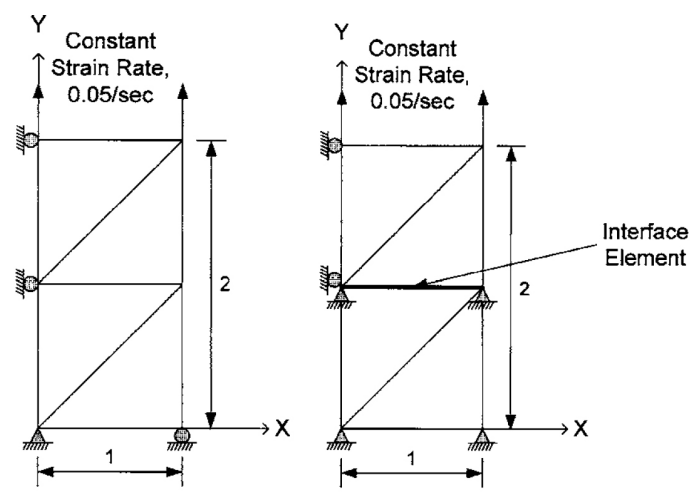

(a) without interface element

(b) with interface element

Figure 4. Four-element uniaxial bar (a) without and (b) with cohesive zone interface element.

$$
\left\{f_{5}^{e}\right\}=-\int_{S_{c}}[N]^{T}\left[\Delta T^{R}\right] d S
$$

In the above equations, $\left[K^{e}\right]=$ element stiffness matrix including the effects from cohesive zone elements; and $\left\{f_{1}^{e}\right\}$, $\left\{f_{2}^{e}\right\},\left\{f_{3}^{e}\right\},\left\{f_{4}^{e}\right\}$, and $\left\{f_{5}^{e}\right\}=$ contributions to the element force vector due to externally applied surface tractions [Equation (8)], stresses at the previous time step [Equation (9)], residual stresses during the time step [Equation (10)], cohesive zone tractions at the previous time step [Equation (11)], and residual cohesive zone tractions during the time step [Equation (12)], respectively. The second term in the element stiffness matrix and force vector terms, $\left\{f_{4}^{e}\right\}$ and $\left\{f_{5}^{e}\right\}$, are excluded if the cohesive zone elements are not specified in the body. The terms $\left\{f_{3}^{e}\right\}$ and $\left\{f_{5}^{e}\right\}$ represent viscoelastic characteristics from viscoelastic solid elements (asphalt matrix) and viscoelastic cohesive zone elements (asphalt mastic), respectively. After assembling every element, the total global system of equations can be obtained.

\section{Validation of Finite-Element Code}

The finite-element code developed in this study can be validated by simply comparing computational results from the finite-element code to analytical results obtained from simple problems that are easily solvable. The problems to be analyzed for verifying the finite-element code are shown in Figure 4. The first problem, a simple linear viscoelastic four-element uniaxial bar illustrated in Figure 4(a), was analyzed in an attempt to verify the finite-element code wherein the material viscoelasticity is included without damage. The simulation was performed at a constant strain rate of $0.05 / \mathrm{s}$ with viscoelastic material properties shown in Table 1. A relatively simple and arbitrarily determined linear viscoelastic stress relaxation modulus was used, since this problem is merely for code validation. Analytical linear viscoelastic stresses can be calculated and compared to the computational results from the finite-element analysis. As shown in Figure 5(a), the finite-element prediction and analytical solution are identical. Good agreement between the two results infers that the finite-element code was developed appropriately in terms of material viscoelasticity.

The second problem, a simple linear elastic four-element uniaxial bar demonstrated in Figure 4(b), was analyzed to verify the finite-element code when the viscoelastic cohesive zone is implemented to predict damage development. As can be seen in Figure $4(\mathrm{~b})$, the cohesive zones are modeled by
Table I. Model Properties for Code Validation

\begin{tabular}{lcc}
\hline Parameter & Model properties \\
\hline$v$ & (a) Elastic element & 0.15 \\
$E$ & 55.20 \\
& (b) Viscoelastic element (solid and interface) \\
$v$ & 0.40 \\
$E \infty$ & $1,000.00$ \\
$E 1$ & $4,000.00$ \\
$\eta 1$ & $4,000.00$ \\
& \\
$\delta\left(\delta_{n}=\delta_{t}\right)$ & (c) Interface element (fracture parameters) \\
$r^{0}$ & $1.0000000^{\mathrm{a}}$ \\
$r_{\text {cr }}$ & 0.0002000 \\
$\zeta^{-}$ & 0.0001154 \\
\hline
\end{tabular}

a Arbitrarily assumed for validation.

employing an interface element between two solid elements. In Figure 4(b), a horizontal interface element is placed between two triangular elastic solid elements, and cohesive zones are employed at both ends of the interface element. A uniaxial strain rate of $0.05 / \mathrm{s}$ is applied at the top of the bar for the damage-induced simulation. Elastic properties for the triangular solid elements and viscoelastic properties for the cohesive zone interface element are given in Table 1.

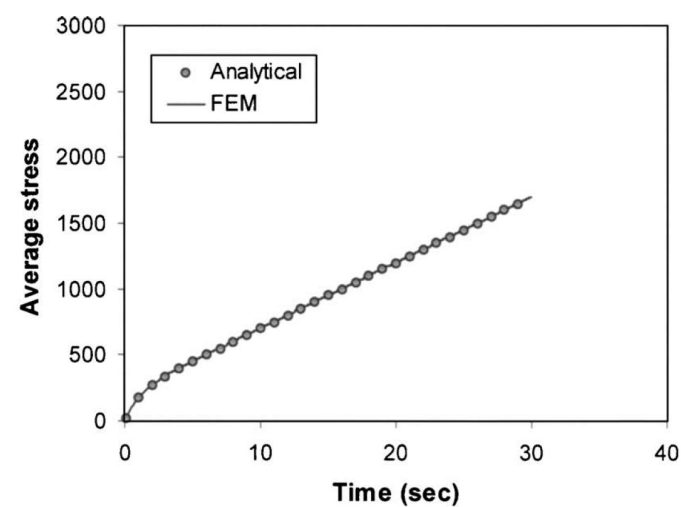

(a)

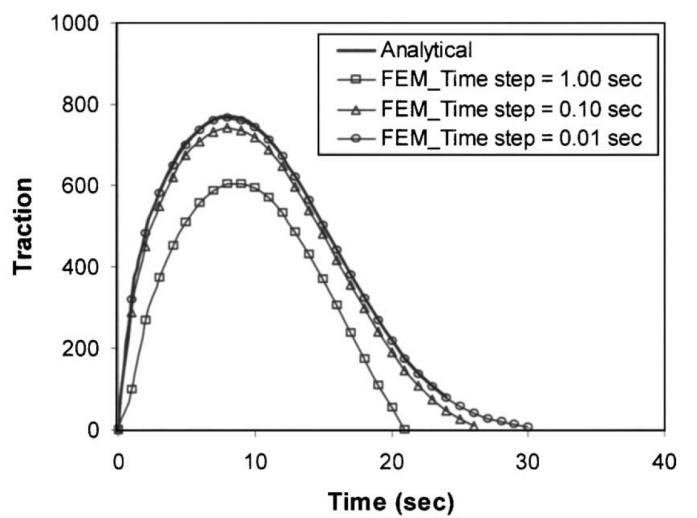

(b)

Figure 5. Comparison between analytical solutions and finite-element analysis results to validate code in terms of (a) material viscoelasticity; (b) cohesive zone damage and failure. 


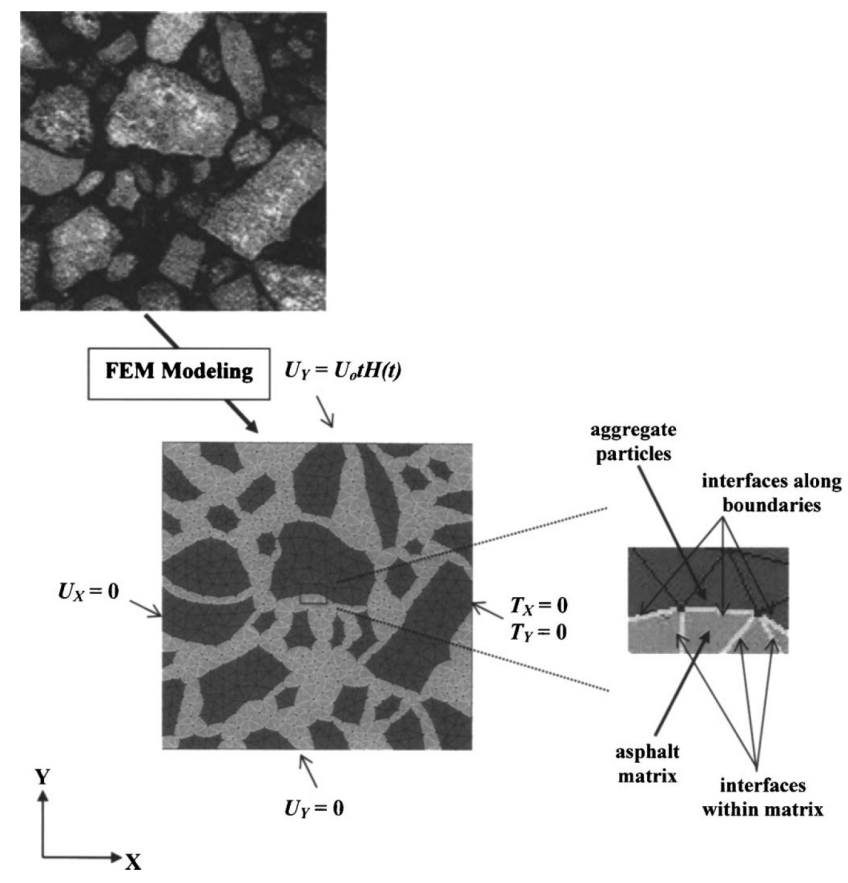

Figure 6. Asphalt concrete sample and its finite-element modeling.

Fracture properties (average values of initial and critical fibril radii, time-independent standard deviation of fibril radii, and cohesive zone material length parameters) used to define the probabilistic damage evolution function, $\alpha(t)$, are also shown in Table 1. Analytical cohesive zone tractions due to nodal displacements can be calculated and compared to computational results. Verification of the code can be confirmed by matching the computational finite-element simulation with the analytical solutions. Figure 5(b) demonstrates that the finite-element predictions and the analytical solutions converge as the time step is reduced. It can be concluded that the damage-induced finite-element code has been developed appropriately.

\section{Finite-Element Model for Asphalt Concrete}

Figure 6 illustrates a digital image of an asphalt concrete mixture $(0.05 \mathrm{~m}$ by $0.05 \mathrm{~m})$ and its finite-element mesh. As can be seen in Figure 6, the asphalt mixture for finite-element modeling exhibits two distinct phases: coarse aggregate particles and a black asphalt matrix phase that is a composite of asphalt cement, fine aggregates, mineral fillers, and air voids. It is widely accepted that damage and fracture of the overall asphalt concrete mixture are predominately impacted by the quality of the asphalt matrix phase, especially at room temperature. The damage initiates with microdamage such as microcracking in the matrix. Based on this fact, the present writers (Kim et al. 2005) modeled damage evolution in the asphalt matrix, in a previous study, by introducing an elastic particle phase (Phase No. 1) signifying fine aggregate and nonlinear viscoelastic interfaces (Phase No. 2) surrounding the particles signifying the asphalt mastic. They measured material properties and fracture properties of the asphalt mastic and incorporated the measured fundamental properties into the finite-element model.

This study models heterogeneous asphalt concrete mixtures with three phases: coarse aggregate particles, the surrounding black phase or asphalt matrix, and the cohesive zone phase

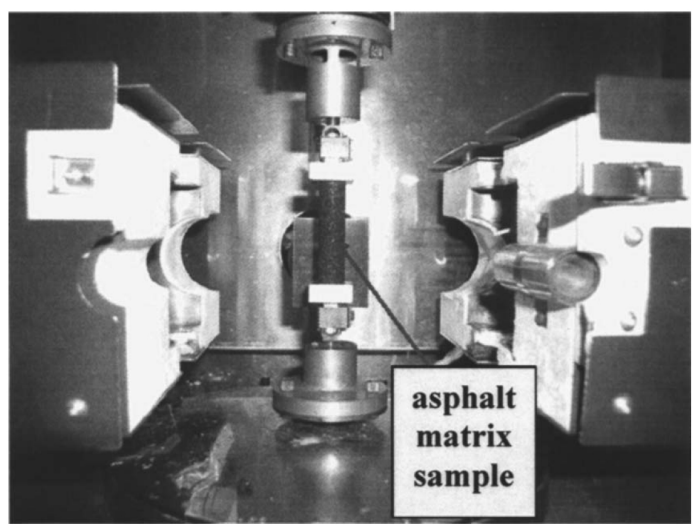

Figure 7. Compacted asphalt matrix sample mounted in the testing instrument to estimate linear viscoelastic material properties.

within the asphalt matrix and along matrix/particle boundaries to signify damage evolution due to crack growth. Coarse aggregate particles and the surrounding asphalt matrix were modeled as isotropic elastic [Equation (1)] and isotropic linear viscoelastic [Equation (2)], respectively. The cohesive zones, which are subjected to damage and fracture due to cracking, were modeled by employing interface elements, as shown in Figure 6. A constitutive law governing mechanical behavior of the cohesive zone is represented by the traction-displacement relation in conjunction with damage evolution characteristics, as presented in Equations (3) and (5). A number of interface elements were randomly oriented in the sample to simulate crack initiation and propagation. Nonlinear viscoelastic damage and failure of the sample due to cracking are modeled by the third phase, cohesive zone interface elements, while coarse aggregate particles and the surrounding asphalt matrix phase are modeled as linear elastic and linear viscoelastic, respectively.

Figure 6 also shows boundary conditions imposed on the mesh. A constant uniaxial tensile displacement rate $U_{Y}=U_{0}$ $t H(t)$ is applied to the top face. The constant $U_{0}=$ unit displacement and $H(t)=$ Heaviside step function. Vertical displacements at the bottom face and horizontal displacements at the left side of the sample are constrained. The creation of a new boundary within the sample due to cracking is monitored through the cohesive zone interface elements in accordance with Equation (3).

\section{Model Properties of Asphalt Concrete}

As noted in Equations (1)-(5), fundamental material properties of each phase (linear elastic coarse aggregate particles, linear viscoelastic asphalt matrix, and linear viscoelastic cohesive zone interfaces), and cohesive zone damage/fracture properties (average values of initial and critical fibril radii, standard deviation of fibril radii, and cohesive zone material length parameters, etc.) are model inputs required to perform finite-element-based analyses.

An elastic modulus of $55.2 \mathrm{GPa}$ and a Poisson's ratio of 0.15 were used for isotropic elastic material properties for the coarse aggregate particles. Viscoelastic material properties of asphalt matrix were determined by performing dynamic frequency sweep testing. An asphalt mastic was mixed with fine aggregates such as Ottawa sand to form cylindrical asphalt matrix samples 50-mm long with a 12-mm diameter. The compacted asphalt matrix sample was then mounted in the testing 
instrument as shown in Figure 7. To determine linear viscoelastic material properties, a torsional dynamic strain $(0.007 \%)$, which is low enough not to cause damage, was applied to the compacted asphalt matrix sample with varying frequencies from 0.01 to $10 \mathrm{~Hz}$ at several different temperatures (10, 25 , and $40^{\circ} \mathrm{C}$ ). The frequency-temperature superposition concept was applied to obtain a long-term frequency-domain linear viscoelastic master curve of the matrix sample. Data plots relating modulus and frequency require curve fitting to identify the linear viscoelastic properties. Among many curve-fitting techniques, the Prony series representation has been frequently adopted because the Prony series fits the data quite precisely and is more efficient for use in mathematical operations. Prony series representations (Christensen 1982) of storage and loss modulus as a function of frequency are

$$
\begin{gathered}
G^{\prime}(\omega)=G_{\infty}+\sum_{i=1}^{n} \frac{G_{i} \omega^{2} \rho_{i}^{2}}{\omega^{2} \rho_{i}^{2}+1} \\
G^{\prime \prime}(\omega)=\sum_{i=1}^{n} \frac{G_{i} \omega \rho_{i}}{\omega^{2} \rho_{i}^{2}+1}
\end{gathered}
$$

where $G^{\prime}(\omega)=$ storage shear modulus; $G^{\prime \prime}(\omega)=$ loss shear modulus; $G_{\infty}=$ long-time equilibrium shear modulus; $G_{i}=$ regression constants; $\rho_{i}=$ relaxation times; $\omega=$ frequency in radian; and $n=$ number of dashpots in the generalized Maxwell model. The frequency domain master curve was then converted to the time domain relaxation modulus, because the linear viscoelastic constitution [Equation (2)] is represented as a function of stress relaxation modulus. If parameters from a Prony series representation are determined from the dynamic frequency sweep test, static relaxation moduli as a function of time can be determined by the following equation:

$$
G(t)=G_{\infty}+\sum_{i=1}^{n} G_{i} e^{-t / \rho_{i}}
$$

where $G(t)=$ static shear relaxation modulus. As can be seen in Equation 15, the static relaxation modulus is easily formulated by using the material parameters $G_{\infty}, G_{i}$, and $\rho_{i}$, which are determined from linear viscoelastic dynamic frequency sweep testing. A more detailed description of sample fabrication and torsional dynamic testing protocol can be found in a study by Kim et al. (2003).

Similarly, the linear viscoelastic material properties of cohesive zone interfaces (asphalt mastic - not including fine aggregates) were measured using a dynamic shear rheometer (DSR). A disk-shaped DSR specimen (2-mm high with an 8$\mathrm{mm}$ diameter) was placed between two plates of the DSR, and a constant shear stress that is sufficiently small not to cause any nonlinear damage, then applied to determine linear viscoelastic creep behavior. Long-term creep behavior of the specimen was determined by applying the time-temperature superposition concept with individual isothermal creep data at three temperatures: 10,31 , and $40^{\circ} \mathrm{C}$. The individual creep test at each temperature was performed to measure data starting from 0.01 to $1,000 \mathrm{~s}$. A master shear creep curve at $25^{\circ} \mathrm{C}$ was then fitted by a Prony series representation as shown below

$$
J(t)=J_{o}+\sum_{k=1}^{m} J_{k}\left(1-e^{-t / \lambda_{k}}\right)
$$

where $J(t)=$ shear creep compliance; $J_{o}=$ initial compliance; $J_{k}$ $=$ regression constants; $\lambda_{k}=$ retardation time; and $m=$ number of dashpots in the model. It is necessary that the Prony

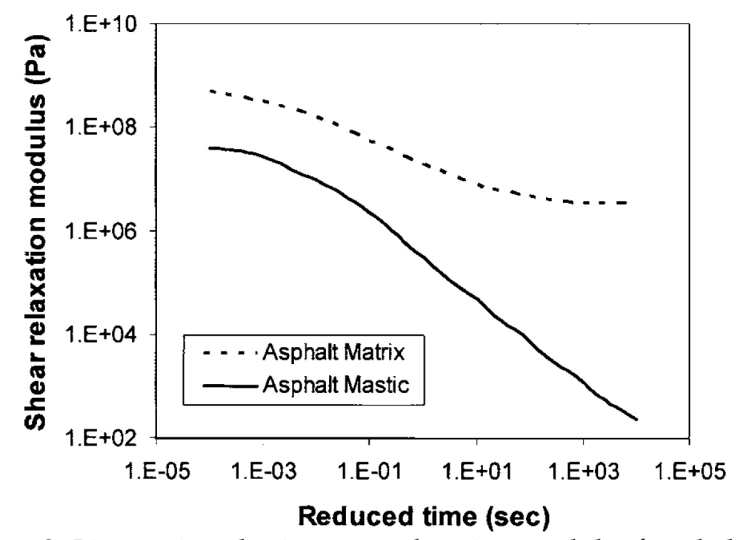

Figure 8. Linear viscoelastic stress relaxation moduli of asphalt mastic and asphalt matrix.

series representation from the DSR creep test be numerically changed to the Prony series relaxation function, because the viscoelastic traction-displacement relation [Equation (3)] in the cohesive zone is represented as a function of stress relaxation modulus. Among several candidate techniques, the Laplace-transform domain was selected in this study to establish the interconversion from compliance to modulus. The wellknown identity between modulus and compliance associated with the Prony series representation in the Laplace domain is

$$
\widetilde{G}(s) \tilde{J}(s)=1
$$

where $s=$ Laplace variable

$$
\widetilde{G}(s)=G_{\infty}+\sum_{i=1}^{n} \frac{s \rho_{i} G_{i}}{s \rho_{i}+1}
$$

and

$$
\tilde{J}(s)=J_{o}+\sum_{k=1}^{m} \frac{J_{k}}{s \lambda_{k}+1}
$$

When the creep function constants $J_{\sigma^{\prime}} J_{k^{\prime}} \lambda_{k}$ are known and the target time constants $\rho_{i}$ are specified, relaxation function constants $G_{\infty}, G_{i}$ in Equation (18) can be determined simply by solving the resulting system of linear algebraic equations. The determined relaxation function constants and the target time constants finally define the viscoelastic relaxation behavior of cohesive zone interfaces (asphalt mastic) through Equation (15). Figure 8 illustrates the resulting shear stress relaxation moduli as a function of time for the asphalt matrix and asphalt mastic, respectively.

Damage/fracture properties of cohesive zone interfaces are characterized by performing fracture tests. In this study, a tensile fracture testing protocol developed by Williams (2001) was used to monitor damage evolution and to define cohesive zone model parameters of the asphalt mastic. Continuous fibril development and cohesive zone growth due to damage were captured using an optical microscope and analyzed by producing video images. The width (or radius) of the fibrils was tracked by pixel counts in the image analysis so that the probabilistic damage evolution function [Equation (5)] could be defined based on the real measurements of initial and critical asphalt fibril radii. The material length parameter can also be obtained from a fracture test, however, exact measurements of the critical displacements at complete separation of the cohesive zone are very difficult with the current fracture testing 
Table 2. Fracture Properties of Asphalt Mastic

\begin{tabular}{cc}
\hline Parameter & $\begin{array}{l}\text { Fracture properties } \\
\text { of asphalt mastic }(\mathrm{m})\end{array}$ \\
\hline$\delta\left(\delta_{n}=\delta_{t}\right)$ & 0.0002308 \\
$r^{0}$ & 0.0002000 \\
$r_{\mathrm{cr}}$ & 0.0001154 \\
$\zeta$ & 0.0000567 \\
\hline
\end{tabular}

device, because film thicknesses of asphalt mastic samples are inevitably thicker (approximately $1 \mathrm{~mm}$ ) than film thicknesses (approximately 10-20 $\mu \mathrm{m}$ ) observed from typical asphalt concrete mixtures. Due to this fact, the material length parameter was determined from a calibration process to match model predictions to actual behavior of global asphalt concrete mixtures. This limitation needs to be overcome so that all required model inputs addressing fracture of the asphalt sample can be directly obtained from the fracture test. Several advanced features including an automatic sample geometry control system are now being installed as an upgrade to the current fracture testing device. Table 2 summarizes the fracture/damage properties of the asphalt mastic used in this study.

It should be noted that only fundamental material properties and fracture properties of each mixture component are necessary inputs in the computational model developed in this study. Therefore, this approach offers time and cost savings in investigating competing material components compared to expensive and repetitive laboratory tests on bulk asphalt concrete samples.

\section{Model Simulations and Discussion}

In this section computational results are presented and compared to laboratory testing data. Efficiency in predicting damage in asphalt concrete mixtures through computational techniques is discussed, and the sensitivity of the mechanical damage response of the asphalt concrete mixtures depending on material properties is also investigated.

Damage-induced computational simulations of asphalt concrete mixtures subjected to monotonic tensile displacement were performed and are presented in Figure 9. In order to investigate the rate-dependent nature of asphalt samples, two different strain rates, $0.0004 / \mathrm{s}$ and $0.0016 / \mathrm{s}$, were applied. As shown in Figure 9, the material response stiffens with

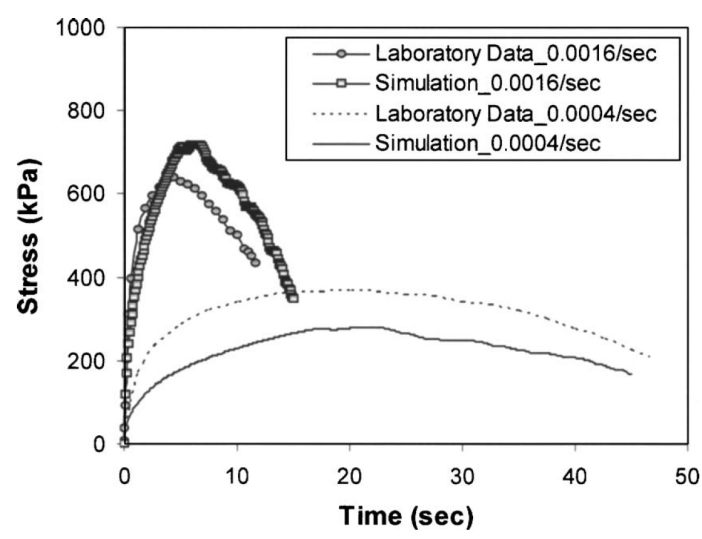

Figure 9. Computational simulations versus laboratory data.

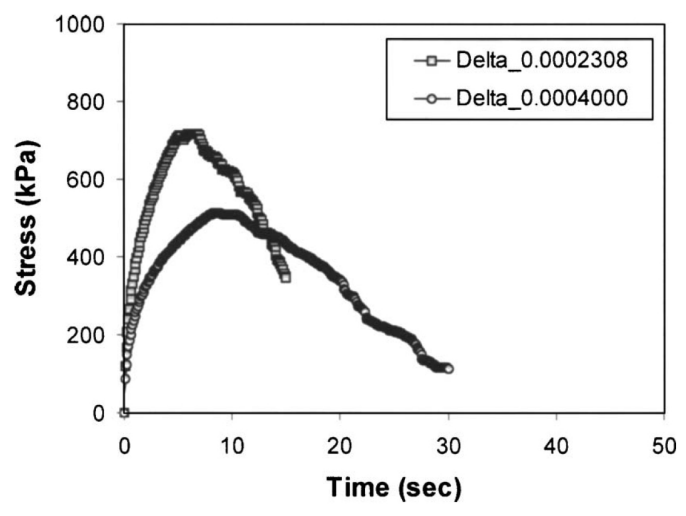

Figure 10. Effect of material length parameter on damaged behavior.

increasing rates of deformation. Figure 9 demonstrates that the deformation rate has a pronounced effect on both the viscoelastic cohesive zones and the viscoelastic solid elements. A slower loading rate produces a more compliant response due to the fact that the slow loading rate allows more time for the viscous mechanisms and microstructural damage in the cohesive zone to occur. Simulation results are generally compatible with laboratory testing results as shown in Figure 9. Both simulations and real testing results clearly demonstrate rate-dependent damage behavior and sample failure that is followed by a significant level of material softening. Even though the finite-element model developed herein showed promising results, the accuracy of the model can be further improved by accounting for several aspects that have not yet been implemented in the model, but such work is in progress. The finite-element mesh used in this study is two-dimensional and was constructed with a digital image $(0.05 \mathrm{~m}$ by $0.05 \mathrm{~m})$ of an asphalt concrete sample. The size of the selected asphalt mixture may not be a representative volume element (RVE) for simulating the overall mechanical behavior of bulk asphalt samples. Determination of the RVE creates significant benefits, since mechanical analyses of any size (large-scale) heterogeneous asphalt mixture can be reasonably converted to mechanical analyses of a smallscale heterogeneous body (typically referred to as the RVE), since the selected RVE scale is sufficient to reflect the overall behavior of the large-scale body. Due to the extremely complicated geometric and mechanical characteristics of asphalt concrete mixtures, the determination of the RVE is complex and must be carefully considered. In addition to the RVE issue, the model developed in this study can be enhanced by considering the effects of coarse aggregate particles in the mixture and plastic and/or viscoplastic behavior in the matrix phase. Rotation of aggregate particles and particle-to-particle contacts should be considered in the computational model. Furthermore, additional constitutive packages such as plastic and/or viscoplastic material packages can be integrated into the model to produce more accurate and comprehensive predictions at a wide range of temperatures and loading conditions. Several studies (Perl et al. 1983; Uzan 1996; Lu and Wright 1998; Seibi et al. 2001; Chehab et al. 2003; Collop et al. 2003; Park 2004; Tashman et al. 2004) have demonstrated the effects of plastic and/or viscoplastic nature in modeling mechanical behavior of asphalt concrete mixtures and asphaltic pavements.

Figures 10-12 present computational predictions that are dependent on specified damage parameters: material length parameter $\left(\delta_{i}\right)$, averaged value of initial fibril radii $\left(r^{0}\right)$, and averaged value of critical fibril radii $\left(r_{\mathrm{cr}}\right)$. Figure 10 generally 


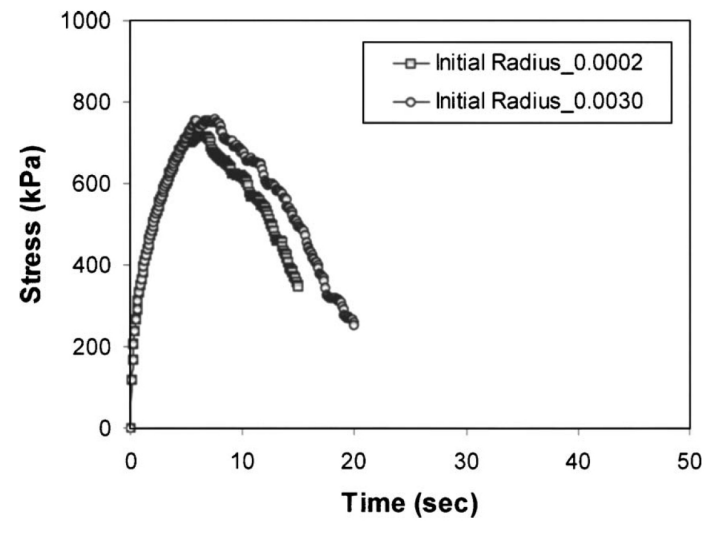

Figure I I. Effect of initial radius on damaged behavior.

demonstrates that the mechanical response is stiffer and the damage growth is faster as the length scale parameter is reduced. This finding is fairly obvious because the lower values of the length scale parameter increase cohesive zone traction $T_{i}$ and cohesive zone strain $\lambda$, correspondingly making the cohesive zone stiffer and damage evolution variable $\alpha(t)$ greater. Figures 11 and 12 illustrate simulation results for different values of averaged initial fibril radius and averaged critical fibril radius, respectively. The amount of damage evolution at a certain time becomes greater as a smaller value of the initial fibril radius is specified, since thinner fibrils approach critical fibril radius faster than thicker fibrils. Similarly, it is readily evident that the damage evolution becomes faster as fibrils reach failure faster, which means that the value of the critical fibril radius is high. More rapid growth of damage contributes to a reduction of resultant tractions in the cohesive zones and a corresponding decrease in average stress of the overall asphalt concrete sample. It appears that the geometric fibril variation of asphalt mastic through the tensile fracture test defines the damage evolution law, and the damage evolution characteristics subsequently predict overall matrix behavior and eventually damage- induced behavior of asphalt concrete mixtures.

The modeling approach presented in this paper forms the basis for practical engineering models for performance analysis and life prediction of asphalt mixtures and pavements based on fundamental principles of mechanics. Since the computational model used in this study accounts for material heterogeneity and geometric complexity due to embedded aggregate particles, inelastic mechanical behavior of the asphalt phase, and rate-dependent crack-associated fracture of the asphalt binders/mastics, a model of this type could strongly impact pavement design and analysis not only by reducing experimental costs significantly, but also by improving the design of mixtures (asphalt concrete mixtures). Furthermore, a computational constitutive modeling technique such as this based on experimental characterization of mechanical properties of each mixture constituent can be successfully applied to many other geomaterials and geostructures such as soil-aggregate systems, soil-structure interactions, porous media, various types of geosynthetic composites, etc., by appropriately incorporating their constitutive behavior and damage behavior into the model.

\section{Concluding Remarks}

A damage-dependent finite-element constitutive model has been formulated and simulated herein for analysis of as-

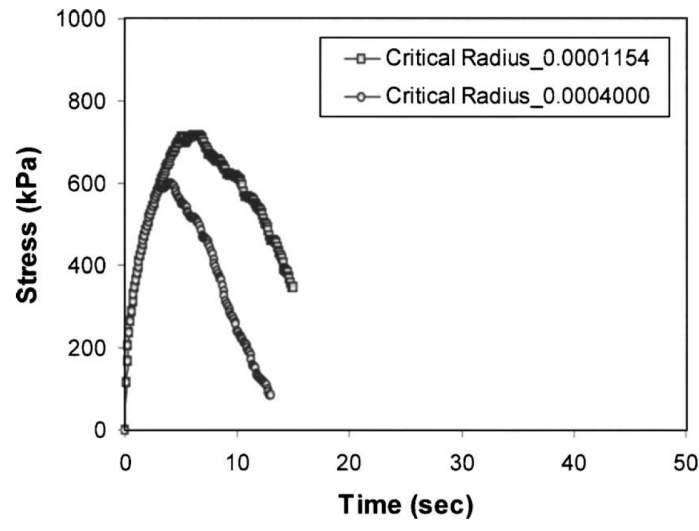

Figure I2. Effect of critical radius on damaged behavior.

phalt concrete mixtures that are composed of elastic aggregate particles, a viscoelastic matrix, and viscoelastic cohesive zones that are subjected to damage due to cracking. Heterogeneous geometric characteristics and inelastic mechanical behavior of asphalt concrete mixtures are successfully taken into account by employing computational modeling and viscoelastic material modeling. Crack growth due to damage evolution is characterized by a nonlinear viscoelastic model and a fracture test-based probabilistic damage evolution law. The locally measured small-scale fracture properties based on simple fracture tests appear to indeed be capable of predicting microscale damage growth due to microcracking, material softening after peak stress, macrocracking due to coalescence of microcracks, and eventual sample failure. The model presented provides a substantial advantage, since the model only requires small-scale fracture/damage properties and fundamental material properties of each mixture component to predict macroscale damage and failure of complex global-scale heterogeneous mixtures. This approach can significantly save time and cost associated with expensive laboratory tests.

Although promising results were obtained in this study, several important follow-up studies to improve the model must continue. The modeling in this study primarily addresses the nonlinear viscoelastic damage in the matrix phase due to the fact that most damage in asphalt concrete mixtures typically initiates in the matrix phase at intermediate temperatures or lower. However, as mentioned earlier, effects of coarse aggregate particles, effects of preexisting defects such as air voids, and plastic-viscoplastic behavior in the mixture should be considered in order to more accurately predict damage-induced behavior of asphalt concrete mixtures. The ability of this model to predict damage will also be improved by taking into account two distinct cohesive zone constitutive relations (or model parameters) for each different fracture mode separately: fracture within the asphalt mastic and fracture along mastic-aggregate boundaries. One possible way to do this is to introduce surface energy characteristics of each mixture constituent, since mechanical bonding within the asphalt-aggregate system is directly related to the surface energy of the mixture constituents (Cheng et al., 2003). It is also imperative to establish the appropriate representative volume element in order to increase predicting accuracy and modeling efficiency.

\section{Acknowledgments}

The writers would like to acknowledge and are grateful for the asphalt concrete testing data provided by Dr. H. Lee at Sejong University in Korea. 


\section{References}

Allen, D. H., and Searcy, C. R. (2000). "Numerical aspects of a micromechanical model of a cohesive zone." J. Reinf. Plast. Compos., 19:3, 240-248. Allen, D. H., and Searcy, C. R. (2001a). "A micromechanical model for a viscoelastic cohesive zone." Int. J. Fract., 107:2, 159-176.

Allen, D. H., and Searcy, C. R. (2001b). “A micromechanically based model for predicting dynamic damage evolution in ductile polymers." Mech. Mater., 33:3, 177-184.

Barenblatt, G. I. (1962). "The mathematical theory of equilibrium cracks in brittle fracture." Adv. Appl. Mech., 7, 55-129.

Birgisson, B., Sangpetngam, B., and Roque, R. (2002). "Predicting viscoelastic response and crack growth in asphalt mixtures with the boundary element method." Transportation Research Record. 1789, Transportation Research Board, Washington, D.C., 129-135.

Chehab, G., Kim, Y. R., Schapery, R. A., Witczak, M. W., and Bonaquist, R. (2003). "Characterization of asphalt concrete in uniaxial tension using a viscoelastoplastic continuum damage model." Asph. Paving Technol., 72, 315-355.

Cheng, D., Little, D. N., Lytton, R. L., and Holste, J. C. (2003). "Moisture damage evaluation of asphalt mixtures by considering both moisture diffusion and repeated-load conditions." Transportation Research Record. 1832, Transportation Research Board, Washington, D.C., 42-49.

Christensen, R. M. (1982). Theory of viscoelasticity: An introduction, Academic, New York.

Collop, A. C., Scarpas, A., Kasbergen, C., and Bondt, A. D. (2003). “Development and finite-element implementation of stress-dependent elastoviscoplastic constitutive model with damage for asphalt." Transportation Research Record. 1832, Transportation Research Board, Washington, D.C., 96-104.

Daniel, J. S., and Kim, Y. R. (2002). “Development of a simplified fatigue test and analysis procedure using a viscoelastic, continuum damage model." Asph. Paving Technol., 71, 619-650.

Dugdale, D. S. (1960). "Yielding of steel sheets containing slits." J. Mech. Phys. Solids, 8, 100-104.

Gibson, N. H., Schwartz, C. W., Schapery, R. A., and Witczak, M. W. (2003). "Viscoelastic, viscoplastic, and damage modeling of asphalt concrete in unconfined compression." Transportation Research Record. 1860, Transportation Research Board, Washington, D.C., 3-15.

Guddati, M. N., Feng, Z., and Kim, Y. R. (2002). "Towards a micromechanics-based procedure to characterize fatigue performance of asphalt concrete." Transportation Research Record. 1789, Transportation Research Board, Washington, D.C., 121-128.

Kim, Y., Little, D. N., and Lytton, R. L. (2002). “Use of dynamic mechanical analysis DMA. to evaluate the fatigue and healing potential of asphalt binders in sand asphalt mixtures." Asph. Paving Technol., 71, 176-206.

Kim, Y., Little, D. N., and Lytton, R. L. (2003). "Fatigue and healing characterization of asphalt mixtures." J. Mater. Civ. Eng., 15:1, 75-83.

Kim, Y., Allen, D. H., and Little, D. N. (2005). “Damage-induced modeling of asphalt mixtures through computational micromechanics and cohesive zone fracture." J. Mater. Civ. Eng., 17:5, 477-484.
Lee, H. J., Daniel, J. S., and Kim, Y. R. (2000). “Continuum damage mechanics-based fatigue model of asphalt concrete." J. Mater. Civ. Eng., 12:2, 105-112.

Lee, H. J., Kim, Y. R., and Lee, S. W. (2003). “Prediction of asphalt mix fatigue life with viscoelastic material properties." Transportation Research Record. 1832, Transportation Research Board, Washington, D.C., 139-147.

Lu, Y., and Wright, P. (1998). "Numerical approach of viscoelastoplastic analysis for asphalt mixtures." Comput. Struct., 69:2, 139-147.

Masad, E., Tashman, L., and Little, D. N. (2002). “A continuum damage framework to validate a unified method of aggregate classification based on image analysis." Proc., 10th Annual ICAR Symp., Baltimore.

Needleman, A. (1987). "A continuum model for void nucleation by inclusion debonding." J. Appl. Mech., 54:3, 525-531.

Papagiannakis, A. T., Abbas, A., and Masad, E. (2002). “Micromechanical analysis of viscoelastic properties of asphalt concretes." Transportation Research Record. 1789, Transportation Research Board, Washington, D.C., 113-120.

Park, D. (2004). "Characterization of permanent deformation in asphalt concrete using a laboratory method and an elastic-viscoplastic model." Ph.D. dissertation, Texas A\&M Univ., College Station, Tex.

Park, S. W., Kim, Y. R., and Schapery, R. A. (1996). “A viscoelastic continuum damage model and its application to uniaxial behavior of asphalt concrete." Mech. Mater., 24:4, 241-255.

Perl, M., Uzan, J., and Sides, A. (1983). “Visco-elasto-plastic constitutive law for a bituminous mixture under repeated loading." Transportation Research Record. 911, Transportation Research Board, Washington, D.C., 20-27.

Sadd, M. H., Dai, Q., Parameswaran, V., and Shukla, A. (2003). “Simulation of asphalt materials using a finite-element micromechanical model with damage mechanics." Transportation Research Record. 1832, Transportation Research Board, Washington, D.C., 86-95.

Seibi, A. C., Sharma, M. G., Ali, G. A., and Kenis, W. J. (2001). “Constitutive relations for asphalt concrete under high rates of loading." Transportation Research Record. 1767, Transportation Research Board, Washington, D.C., 111-119.

Soares, B. J., Freitas, F., and Allen, D. H. (2003). “Crack modeling of asphaltic mixtures considering heterogeneity of the material." Transportation Research Record. 1832, Transportation Research Board, Washington, D.C., 113-120.

Tashman, L., Masad, E., Little, D. N., and Lytton, R. L. (2004). “Damage evolution in triaxial compression tests of HMA at high temperatures." Asphalt Paving Technol., 73, 53-87.

Tvergaard, V. (1990). "Effect of fiber debonding in a whisker-reinforced metal." Mater. Sci. Eng., A, 125:2, 203-213.

Uzan, J. (1996). "Asphalt concrete characterization for pavement performance prediction.” Asph. Paving Technol., 65, 573-607.

Williams, J. J. (2001). "Two experiments for measuring specific viscoelastic cohesive zone parameters." Master's thesis, Texas A\&M Univ., College Station, Tex.

Zocher, M. A., Allen, D. H., and Groves, S. E. (1997). “A three- dimensional finite-element formulation for thermoviscoelastic orthotropic media." Int. J. Numer. Methods Eng., 40:12, 2267-2288. 